\title{
Calculation of an Optical setup for a
}

\section{LIBS system}

DOI: $10.30609 / J E T I .2018-1.5163$

\section{Thalena C. Zanetti ${ }^{1}$, Jader S. Cabral ${ }^{1 *}$}

${ }^{1}$ Instituto de Física, Universidade Federal de Uberlândia, P.O. Box 593, 38400-902, Uberlândia, MG, Brazil.

\begin{abstract}
In this work we will represent the calculation for an optical LIBS system, as well as the parameters for the beam characterization. On the realized simulation, the beam was treated with the wave optics theory and its propagation with the paraxial approximation. It was described as a beam of Gaussian intensity profile and its propagation was acquired through the ABCD's law. Our results have showed the appropriated lens to hold a LIBS sign for different kinds of materials, depending on their bond cleavage. Also, we have showed a table with typical values of focus lens, used in LIBS setups, and its respective irradiance.
\end{abstract}

Key words: Spectroscopy, LIBS, Gaussian beam. 


\section{1.- Introduction}

The LIBS (Laser Induced Breakdown Spectroscopy) system is widely used in several research fields for atomic analysis of many materials, including alloys, soils, fertilizers, grains, architectonic objects, semiconductors and medicines, among others [1-7]. A short period of time have passed since the first experiments, and the technique progress is still far away from stagnate. The fact of LIBS system owns a great potential as an analytical tool originated a kind interest by the scientific community in the last decades. As a result, the number of international publications related with the technique has grown substantially. In 2011, a LIBS system was coupled to the Curiosity robot and then it was sent to Mars, showing its maturity and utility $[8,9]$.

The LIBS technic is a type of atomic emission spectrometry that uses the plasma generation by high-power short pulses of a laser. The laser's pulse duration is typically of nanoseconds (ns), however there are experiments with lasers of picoseconds (ps) and femtoseconds (fs) [2,10-12]. The high-power laser focusing in the sample $\left(\sim \mathrm{GW} / \mathrm{cm}^{2}\right)$ causes ablation of a small portion of matter, in order of nano grams, which generates a plasma plumb with a temperature over $50.000 \mathrm{~K}$. In this temperature, the material dissociates into ions and excite atoms, emitting a continuous spectrum of radiation, which is not useful to characterize materials. Due to the high velocity of plasma elements, occurs a supersonic and adiabatic expansion, cooling the plasma between $5.000 \mathrm{~K}$ and $15.000 \mathrm{~K}$ in approximately 1 to $2 \mu \mathrm{s}$, when it is possible to measure the atomic/ionic emission lines and identify the elements present in the sample. The time between the ablation pulse and the radiation acquirement can vary in the range of 1 to $10 \mu \mathrm{s}$.

Owing the small amount of material which is wasting in the analysis (ng and fg), LIBS is considered a semi-destructive technique for several applications, since with an average power density less than $1 \mathrm{~W}$, the sample don't heat out of ablation region. Because of its nature, this technique enables in loco analysis, which deals out complex sample preparation processes, eliminating production of chemistry residues [13-14]. Other possibility is to use it in gaseous, liquid or solid samples, in conductor and non-conductor materials and also on that hardly dissolution [15]. 
In this work we present the calculation achieved for an optical LIBS system setup supposing the Gaussian profile of the laser beam. To do so, we applied the Maxwell's Equations resolution in cylindrical coordinates and the Gaussian beam propagation method in optical systems. Thereby, we have found the best optical setup which will allow us the suitable power density $\left(\mathrm{Gw} / \mathrm{cm}^{2}\right)$ to reach the breakdown of different sample matrixes.

\section{2.- Gaussian beam and its propagation}

From Maxwell's four equations, that describe wave light behavior, we can derive the wave equation for electric field $\vec{E}$, given by eq. (1):

$$
\nabla^{2} \vec{E}+k^{2}(\vec{r}) \vec{E}=0
$$

where $k=\mu \epsilon \omega^{2}$ is the wave vector, $\mu$ is the magnetic permeability of the medium, $\epsilon$ is the electric permittivity and $\omega$ is the wave frequency. For simply, we'll treat the case in which the medium is homogeneous and non-magnetic, i. e., the vector $\vec{k}$ is a constant.

Writing the laplacian operator in cylindrical coordinates and separating it into transverse portion to beam propagation $(r, \theta)$ and parallel to beam propagation $(z)$, we achieved the wave equation resolution e we found the electric field expression $[16,17]$.

As the wave intensity is proportional to the square of electric field magnitude, thus the light intensity $I(r, z)$, resultant from $\vec{E}$ wave equation, is given by eq. (2) and exhibits the Gaussian form, with maximum intensity $I_{0}\left(\frac{w_{0}}{w(z)}\right)^{2}$ at radial coordinate $r=0$.

$$
I(r, z)=I_{0}\left(\frac{w_{0}}{w(z)}\right)^{2} e^{-2 \frac{r^{2}}{w^{2}(z)}}
$$

For $r=w(z)$, the beam intensity decreases $1 / e^{2}$ from its maximum value and this distance is called beam ray. Then the parameter $w_{0}$ is called waist and its 
relation with $w(z)$ is given by eq. (3). Note that, in the origin of beam propagation $z=0$, the beam reaches its minimum value $w_{0}$.

$$
w^{2}(z)=w_{0}^{2}\left\{1+\left(\frac{z}{z_{0}}\right)^{2}\right\}
$$

The parameter $z_{0}=\frac{k w_{0}^{2}}{2}=\frac{\pi n w_{0}^{2}}{\lambda}$ is called Rayleigh's length and represents the beam focusing region, where $w_{0}<w(z)<\sqrt{2} w_{0}$. For small $z_{0}$, there is a short focalization and for great $z_{0}$, there is a long focalization. Figure 1 illustrates the Gaussian profile of a laser beam.

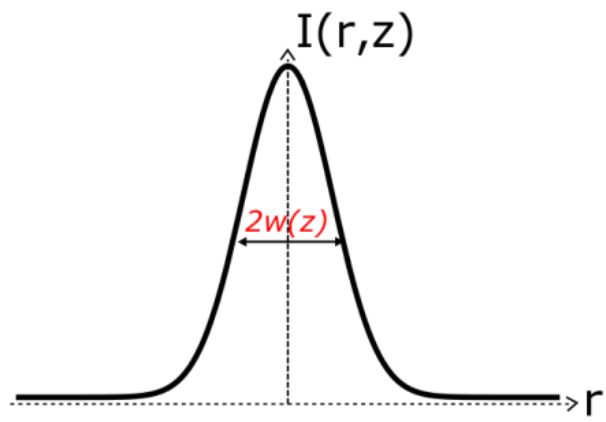

Figure 1: Gaussian profile for a laser beam.

To find the beam ray $w(z)$, the waist $w_{0}$ and the curvature ray of the wavefront $R(z)$ as the beam propagates and interacts with the optical elements (mirrors and lens), we need to apply the ABCD's law, widely used in geometric optics and that, with the correct construction of parameters which describe the beam, can be utilized in the Gaussian wave [17]. To do so, it is defined the parameter $q(z)$, given by eq. (4), which is a complex number. Note that its real part gives information about the beam wavefront $R(z)$ and its imaginary part depends on the beam ray $w(z)$.

$$
\frac{1}{q(z)}=\frac{1}{R(z)}-\frac{i \lambda}{\pi n w^{2}(z)}
$$


where $n$ is the refraction index of the propagation medium and $\lambda$ is the wavelength in the vacuum.

In accord with the matrix optics, each optical element can be represented by a unitary matrix $2 x 2$ and its elements are called A, B, C and D in the form $\left(\begin{array}{ll}A & B \\ C & D\end{array}\right)$. When the beam strikes an optical element, the new parameter $q_{2}(z)$ is given by eq. (5) and thus it is possible to find a new beam ray $w(z)$ and the new wavefront $R(z)$.

$$
q_{2}(z)=\frac{A q(z)+B}{C q(z)+D}
$$

In this way, in an optical system composed of various elements, we only need to apply this routine in each element or to calculate the total matrix of the optical system, given by the multiplication of individual matrixes, to characterize the Gaussian beam, i. e., to find its ray $w(z)$ and the ray of the wavefront $R(z)$.

\section{3.- Calculation for the optical LIBS system setup}

To determine the best optical setup of a LIBS system, we have solved the following problem: assuming the beam arrives collimated at the focusing lens of the LIBS system, which is the beam waist $w_{0}$ after the lens. Besides, we need to answer if the joined $w_{0}$ is enough to presents a power density (irradiance) able to cause the material ablation and to create the LIBS signal. Figure 2 illustrates the calculated situation.

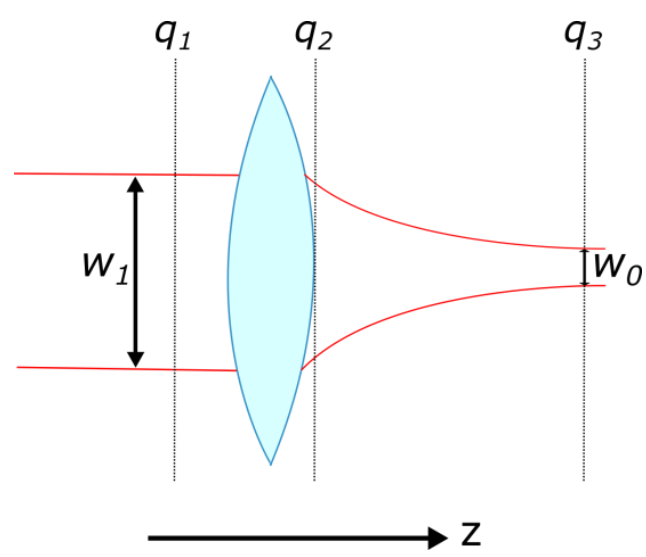

Figure 2: Optical setup of the LIBS assembly. 
As the beam is collimated when strikes the lens, its wavefront ray $R_{1} \rightarrow \infty$. Thus, by eq. (4), we have $\frac{1}{q_{1}}=-i \frac{\lambda}{n \pi w_{1}^{2}}$. Using ABCD's law, eq.(5), and a thin lens matrix $\mathcal{M}_{\text {LENTE }}=\left(\begin{array}{cc}1 & 0 \\ -1 / f & 1\end{array}\right)$, we obtain that the parameter $q_{2}$, immediately after the lens, satisfies the following relation: $\frac{1}{q_{2}}=\frac{-\frac{1}{f}+i \frac{\lambda}{n \pi w_{1}^{2}}}{\left(-\frac{1}{f}\right)^{2}+\left(\frac{\lambda}{n \pi w_{1}^{2}}\right)^{2}}$, where $f$ is the lens focusing distance.

After the lens, we apply the ABCD's law once more, eq. (5), for a translation with distance $l$, whose matrix is $\mathcal{M}_{\text {TRANSL }}=\left(\begin{array}{ll}1 & l \\ 0 & 1\end{array}\right)$ and we obtain that the parameter $q_{3}$ satisfies the relation given by eq. (6).

$$
\frac{1}{q_{3}}=\frac{\left(a^{2}+b^{2}\right)\left[l\left(a^{2}+b^{2}\right)-a-i b\right]}{\left[l\left(a^{2}+b^{2}\right)-a\right]^{2}+b^{2}}
$$

where we introduce the parameters $a=\frac{1}{f}, b=\frac{\lambda}{n \pi w_{1}^{2}}=\frac{1}{z_{01}}$ to simplify the equation.

As Figure 2 shows, at $q_{3}$ plan the wavefront ray is $R_{3} \rightarrow \infty$, therefore the real part of eq. (6) is null. This information takes us to find the translation distance $l$ as a function of the lens focusing distance $f$ and the Rayleigh's length $z_{01}$, eq. (7).

$$
l=\frac{f}{1+\left(\frac{f}{z_{01}}\right)^{2}}
$$

Note that, if $z_{01} \gg f$, we have $l=f$, i. e., we are at geometric optics regime. Matching the imaginary part of eq. (6) with imaginary part of eq. (4), we have:

$$
\frac{\left(a^{2}+b^{2}\right) b}{\left[l\left(a^{2}+b^{2}\right)-a\right]^{2}+b^{2}}=\frac{\lambda}{\pi n w_{0}^{2}}
$$

Substituting the values of $a$ and $b$ in the equation above, we achieved the relation between waist $w_{0}$ and its initial ray $w_{1}$, given by eq. (9).

$$
w_{0}=\frac{f}{z_{01}{\sqrt{1+\left(\frac{f}{z_{01}}\right)^{2}}}^{2}} \cdot w_{1}
$$


Note that, if the Rayleigh's length $z_{01}$ is larger than the lens focusing distance $f$, eq. (9) will assume the simple form $w_{0}=\frac{f \lambda}{n \pi w_{1}}$.

To represent a LIBS system, we use a pulsed Nd:YAG laser, with pulse width of 10 nanoseconds, in fundamental harmonics $(\lambda=1064 \mathrm{~nm})$ and energy about 50 mJ per pulse. Figure 3 shows the variation of the lens focusing distance value and the calculation of light irradiance at $w_{0}$, i. e., where the analyzed sample was placed. The irradiance calculation was achieved dividing the beam power $\left(\frac{50 \mathrm{~mJ}}{10 \mathrm{~ns}}\right)$ by the lighted area $\pi w_{0}^{2}$ in the sample.

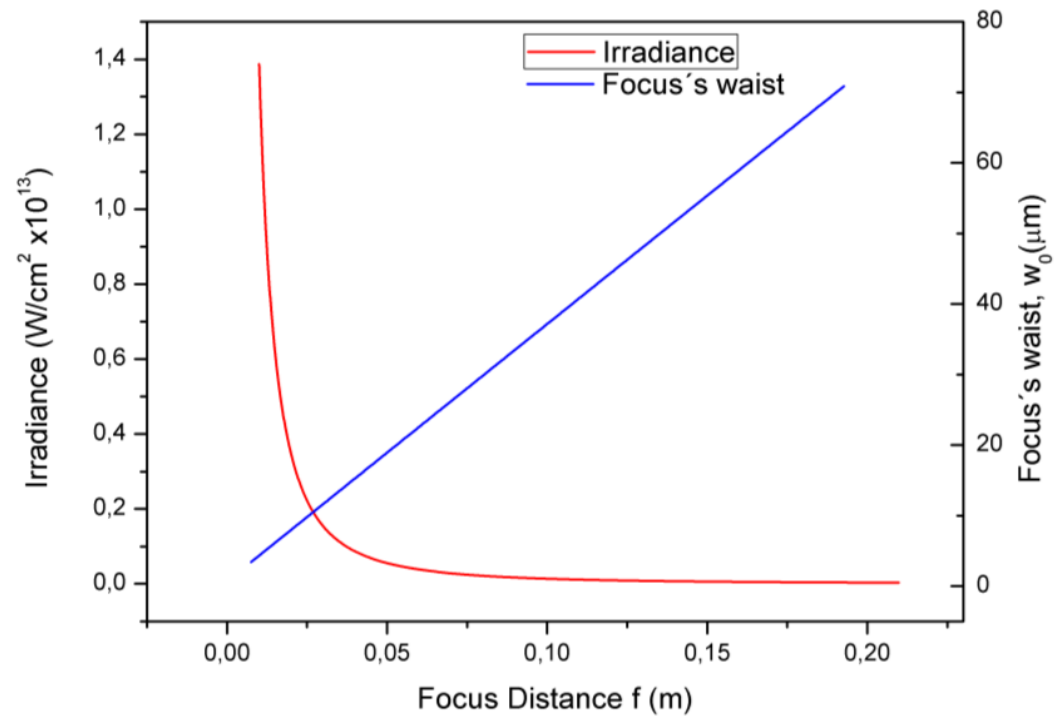

Figure 3: Irradiance and $w_{0}$ versus Focus Distance plot for a LIBS assembly. The $w_{1}$ utilized on simulation was $1 \mathrm{~mm}$, according to actual lasers Nd:YAG.

For sample matrixes utilized in LIBS spectroscopy, bond cleavage following material ablation happened in the range of 5 to $10 \mathrm{GW} / \mathrm{cm}^{2}$. In this case, we can overcome this value with the initial use of a lens with focusing distance of to $20 \mathrm{~cm}$. If in the experiment it was necessary a higher or lesser irradiance in the sample, we would change it easily according to the calculation realized and the Figure 3. In Table 1, we show the values of $w_{0}$ and irradiance for some $f$ values usually used in LIBS assemblies. 
Table 1. $w_{0}$ and Irradiance values for focus distances usually used in LIBS assemblies.

\begin{tabular}{|c|c|c|}
\hline Focus Distance $\boldsymbol{f}(\mathbf{c m})$ & $\boldsymbol{w}_{\mathbf{0}}(\boldsymbol{\mu m})$ & Irradiance $\left(\mathbf{G W} / \mathbf{c m}^{2}\right)$ \\
\hline 5,0 & 16,9 & $5,55 \times 10^{2}$ \\
\hline 7,5 & 25,4 & $2,46 \times 10^{2}$ \\
\hline 10,0 & 33,8 & $1,39 \times 10^{2}$ \\
\hline 12,5 & 42,3 & $8,90 \times 10^{1}$ \\
\hline 15,0 & 50,7 & $6,18 \times 10^{1}$ \\
\hline 20,0 & 67,6 & $3,48 \times 10^{1}$ \\
\hline
\end{tabular}

\section{4.- Conclusion}

Utilizing the Gaussian beam formalism to treat the laser used in Laser Induced Breakdown Spectroscopy (LIBS) and the ABCD's law to model its propagation in the optical system, it was possible to find the values of focusing distance more suitable for LIBS setup. The simulation was reliable enough to change the optical assembly in the laboratory without compromise its validity. With a lens of $f=7,5 \mathrm{~cm}$ we achieved an irradiance in the sample of approximately $246 \mathrm{GW} / \mathrm{cm}^{2}$, which is enough to cause material ablation and consequently to obtain a LIBS signal.

The realized simulation in this work was necessary to know the beam parameters before and after it strikes the sample. Thus, the optical assembly of the LIBS system has all of its parameters known, that permits more agility and efficiency in the measurements and sample changes.

\section{5.- Bibliography}

1 Fortes, F.J.; Moros, J.; Lucena, P.; Cabalín, L.M.; Laserna, J.J. Laser-induced breakdown spectroscopy. Analytical chemistry, v. 85, n. 2, p. 640-69, 2013.

2 Hahn, D.W.; Omenetto, N. Laser-induced breakdown spectroscopy (LIBS), part II: 
review of instrumental and methodological approaches to material analysis and applications to different fields. Applied spectroscopy, v. 66, n. 4, p. 347-419, 2012.

3 Haddad, J. El; Canioni, L.; Bousquet, B. Good practices in LIBS analysis: Review and advices. Spectrochimica Acta Part B: Atomic Spectroscopy, v. 101, p. 171-182, 2014.

4 Nicolodelli, G; Senesi, G.S.; Romano, R.A.; Cabral, J.; Perazolli, I.L.O; Marangoni, B.S.; Villas-Boas, P.R.; Milori, D.M.B.P. Laser-induced breakdown spectroscopy of envorinmetal and synthetic samples using non-intensified CCD: optimization of the excitation wavelength. Applied Physics B, p. 123-127, 2017.

5 Nicolodelli, G; Senesi, G.S.; Ranulfi, A.C.; Marangoni, B.S.; Watanabe, A.; Benites, V.M.; Oliveira, P.P.A.; Villas-Boas, P.R.; Milori, D.M.B.P. Double-pulse laser induced breakdown spectroscopy in orthogonal beam geometry to enhance line emission intensity from agricultural samples. Microchemical Journal, v. 133, p. 272-278, 2017.

6 Marangoni, B.S; Silva, K.S.G.; Nicolodelli, G.; Senesi, G.S.; Cabral; J.S.; VillasBoas, P.R.; Silva, C.S.; Teixeira, P.C.; Nogueira, A.R.A.; Benites, V.M.; Milori, D.M.B.P. Phosphorus quantification in fertilizers using laser induced breakdown spectroscopy (LIBS): a methodology of analysis to correct physical matrix effects. Analytical Methods, v. 8, p. 78-82, 2016.

7 Nicolodelli, G; Marangoni, B.S.; Cabral, J.S.; Villas-Boas, P.R.; Senesi, G.S.; Santos, C.H.; Romano, R.A.; Segnini, A.; Lucas, Y.; Montes, C.R.; Milori, D.M.B.P. Quantification of total carbon in soil using laser-induced breakdown spectroscopy: a method to correct interference lines. Applied Optics, v. 53, n. 10, p. 2170-2176, 2014.

8 Maurice, S.; Wiens, R.C.; Saccoccio, M.; Barraclough, B.; Gasnault, O.; Forni, O.; Mangold, N.; Baratoux, D.; Bender, S.; et al. The ChemCam Instrument Suite on the Mars Science Laboratory (MSL) Rover: Science Objectives and Mast Unit Description. Space Science Reviews, v. 170, n. 1-4, p. 95-166, 2012.

9 Wiens, R.C.; Maurice, S.; Barraclough, B.; Saccoccio, M.; Barkley, W.C.; Bell, J.F.; Bender, S.; Bernardin, J.; Blaney, D.; et al. The ChemCam Instrument Suite on the Mars Science Laboratory (MSL) Rover: Body Unit and Combined System Tests. Space Science Reviews, v. 170, n. 1-4, p. 167-227, 2012.

10 Haddad, J. El; Canioni, L.; Bousquet, B. Good practices in LIBS analysis: Review 
and advices. Spectrochimica Acta Part B: Atomic Spectroscopy, v. 101, p. 171-182, 2014.

11 Hahn, D.; Omenetto, N. breakdown spectroscopy (LIBS), part I: review of basic diagnostics and plasma-particle interactions: still-challenging issues within the analytical plasma community. Applied spectroscopy, v. 64, n. 12, p. 335-66, 2010.

12 Miziolek, A.W.; Palleschi, V.; Schechter, I. Laser Induced Breakdown Spectroscopy. Cambridge: Cambridge University Press, 2006.

13 Assion, A.; Wollenhaupt, M.; Haag, L.; Mayorov, F.; Sarpe-Tudoran, C.; Winter, M.; Kutschera, U.; Baumert, T. Femtosecond laser-induced-breakdown spectrometry for $\mathrm{Ca} 2+$ analysis of biological samples with high spatial resolution. Applied Physics B, v. 77, n. 4, p. 391-397, 2003.

14 Costa, T.E.M.M. (2008). Detecção de transgênicos em alimentos utilizando a técnica multiplex-PCR.

15 Lazic, V.; Jovićević, S. Laser induced breakdown spectroscopy inside liquids: Processes and analytical aspects. Spectrochimica Acta Part B: Atomic Spectroscopy, v. 101, p. 288-311, 2014.

16 Fowles, G.R. Introduction to Modern Optics. Dover Publications, 1975.

17 Yariv, A. Quantum Electronics. John Wiley \& Sons, Inc., 1975. 